Meta

Journal des traducteurs

Translators' Journal

\title{
L'évolution de la profession de traducteur dans le secteur privé torontois au cours des deux dernières décennies
}

\section{Chaké Tchilinguirian}

Volume 27, numéro 2, juin 1982

URI : https://id.erudit.org/iderudit/004329ar

DOI : https://doi.org/10.7202/004329ar

Aller au sommaire du numéro

Éditeur(s)

Les Presses de l'Université de Montréal

ISSN

0026-0452 (imprimé)

1492-1421 (numérique)

Découvrir la revue

Citer cette note

Tchilinguirian, C. (1982). L'évolution de la profession de traducteur dans le secteur privé torontois au cours des deux dernières décennies. Meta, 27(2), 233-237. https://doi.org/10.7202/004329ar d'utilisation que vous pouvez consulter en ligne. 
Patenaude a été le premier président de l'ATIO à partir de 1967 , à militer, après avoir pris conscience de la situation précaire du traducteur, et à cuvrer pour la reconnaissance de la profession, qui conférerait ainsi au traducteur la dignité pour lui permettre de s'affirmer à bien des égards, surtout vis-à-vis de son employeur ou de ses clients.

Dix ans plus tard, soit en mai 1977, à Montréal, inaugurant le VIII e congrès mondial de la FIT, son président $M$. Pierre-François Caillé lançait son cri de triomphe qui couronnait la lutte de toute une vie: «Nairobi, disait-il, c'est la Victoire, c'est notre victoire à nous traducteurs...», et il annonçait à quelque 600 traducteurs venus de 26 pay's différents, qu'à la $\mathrm{XIX}^{\mathrm{e}}$ conférence générale de l'UNESCO à Nairobi (la capitale du Kenya) en novembre 1976, on avait adopté la recommandation dite Document 19C sur la protection juridique des traducteurs et des traductions et sur les moyens pratiques d'améliorer la condition de ceux qui exercent cette profession. Il ajoutait qu'il appartenait désormais aux associations locales, soit à la STQ et à l'ATIO de représenter à l'Office des professions une demande pour la reconnaissance professionnelle du traducteur.

Or, au début de cette année, soit en février 1980, nous avons appris que l'Office des professions du Québec a rejeté la demande de la STQ, après avoir laissé s'écouler trois ans. $\mathrm{La}$ demande de l'ATIO auprès du gouvernement ontarien est également mise en veilleuse, malgré les relances répétées des présidents qui se sont succédé.

Donc le thème de mon exposé aujourd'hui porte sur l'évolution, ou bien disons sur l'évaluation de l'évolution de la carrière, on pourrait aussi dire du métier ou de la profession de traducteur, dans la région torontoise et dans les villes avoisinantes au cours des deux dernières décennies.

Pour nous situer tout de suite dans la perspective, je dois préciser que toutes les traductions à de rares exceptions près, qui se font dans le secteur privé à Toronto et aussi à $\mathrm{Ha}$ milton, Niagara Falls, Welland, London, Waterloo, etc., sont de caractère technique, administratif ou scientifique et, dans $95 \%$ des cas, elles se font de l'anglais vers le français parce qu'elles sont destinées au marché québécois essentiel-

L'ÉVOLUTION DE LA PROFESSION DE TRADUCTEUR DANS LE SECTEUR PRIVÉ TORONTOIS AU COURS DES DEUX DERNIERES DÉCENNIES*

Si je désire rappeler ici le nom de ces deux timonniers de la profession, c'est que l'un, Paul

* Colloque de Glendon, 1980. lement. En ce début de la révolution tranquille du Québec des années 60, les maisons mères des grosses compagnies, en particulier des compagnies d'assurance et des grands magasins sis à Toronto et faisant affaire au Québec, se sont vues, petit à petit, obligées, pour garder leur clientèle, à tout le moins de correspondre en français, c'est-à-dire de répondre en français aux lettres et aux commandes ou plaintes reçues dans cette langue. 
Comme les lettres se faisaient nombreuses, les employés unilingues anglophones des services aux clients, le premier moment d'affolement passé, après s'être concertés entre eux sur les aléas d'apprendre la langue seconde ou les possibilités de travailler dans cette langue seconde, puisqu'après tout, chacun d'eux comptait dans son bagage scolaire cinq années d'études assidues de la langue française parisienne, décident d'un commun accord de s'adresser à leur plus proche collègue qui semblait bien se débrouiller dans cette langue difficile, parce qu'on l'entendait souvent l'articuler avec aisance au téléphone, ou parce que son nom semblait avoir des consonances "galliques».

Ainsi venait de naître, à quelques exceptions près, le premier traducteur anglais/français du secteur privé, ainsi se créerait le premier poste de traducteur, après l'écoulement d'un bref laps de temps, au cours duquel le volume du courrier reçu et des réponses à envoyer avait fini par obliger l'employé francophone à se décharger des fonctions originelles pour lesquelles on l'avait engagé tout d'abord.

Quelques mois plus tard, nous voyons notre correspondant-traducteur nanti d'une dactylo. Il ou elle (c'est surtout elle, car la traduction est apparemment un domaine à prédominance féminine), elle, donc, écrit à la main ses traductions, remet le manuscrit parfois grandement raturé à la dactylo. Cette dernière se charge de corriger, si elle le peut, toute erreur d'orthographe ou de grammaire qu'elle a repérée... ou cru repérer. Plus le temps passe, et quelques mois en général semblent être un bon critère d'ancienneté, dans ce métier instable et dynamique où l'on verra un roulement de personnel allant d'un seul jour en poste à une semaine et ne dépassant parfois pas un mois, donc, au fil des semaines qui s'écoulent, la dactylo bilingue, forte de sa formation intensive et accélérée et de l'expérience qu'il lui est ainsi permis d'acquérir, puisqu'on lui a recommandé de se reporter au texte anglais original si elle n'arrive pas à bien déchiffrer l'hiéroglyphe de son supérieur direct, la ou le traducteur, traductrice, certains disent même la traducteuse, mot étrange s'il en fût, la dactylo donc engage une discussion sur la tournure traduite, la propriété des mots, récite quelques règles grammaticales et ne voilà-t-il pas qu'elle reçoit une promotion, avec la recommandation chaleureuse de ladite traductrice, heureuse de s'adjoindre une aide précieuse et peu prétentieuse. La dactylo est promue «Junior translator "; elle continue à taper les lettres que lui remet son supérieur hiérarchique, mais elle le décharge désormais des petits travaux insignifiants. Les relations entre les deux proches collègues sont cordiales. Il est bien rare qu'une bisbille quelconque naisse entre les deux proches collègues, parce qu'il y a respect venant de bas en haut (de l'ancienne dactylo vers le traducteur) et une sorte de re- connaissance venant de haut en bas (du traducteur vers la junior translator), puisque le traducteur, ainsi secondé, peut passer à des traductions plus fouillées, se rengorger et parler avec plus d'assurance, dirait-on avec ses collègues anglophones de l'étage qui commencent à le consulter de plus en plus.

Le traducteur à son tour, de cause à effet, est promu, quelque temps après, "Senior translator». Le temps pour lui est arrivé, d'après les actuaires de la compagnie, qui, grâce à leurs rapports directs et suivis, ont eu l'occasion de l'apprécier, tant sur le plan humain que professionnel, ainsi que de l'avis du chef des services de bureau, d'exercer son art nouveau qu'il se plaît de plus à plus à fignoler, à approfondir, autant que faire se peut, dans une époque où à Toronto il n'existait même pas de librairie française, d'exercer dis-je son art nouveau et d'entamer la traduction des formulaires réservés au public francophone. Le client exige depuis un certain temps déjà, de ne signer qu'un document rédigé en français; néanmoins, en cas de litige, c'est l'original anglais qui fait foi.

Comme c'est un document contractuel, le traducteur senior (ou principal) doit faire lire sa copie avant de l'envoyer à l'imprimeur anglophone, à l'avocat de la compagnie. Ce dernier est un francophone vivant à Toronto; ou bien, un avocat francophone de Montréal. Si la compagnie ne possède pas cet oiseau rare dans ses locaux à Toronto, la copie est envoyée à Montréal. De là, l'avocat, malgré les distances, communique avec le traducteur, le dialogue s'engage, et le texte revient portant des annotations en rouge, mettant en garde contre les tournures ou expressions inacceptables. Voyant ses phrases cerclées de rouge, le traducteur se morfond quelque peu, se promet de pousser ses recherches, puis il se ressaisit, lit le texte raturé avec son subalterne, fait les changements demandés et envoie le tout à l'imprimeur.

Après deux ou trois lectures d'épreuves, parfois plus parce que le typographe, étant anglophone, a forcément interverti l'ordre des lettres, anglicisé par mégarde des tournures familières, le document est dûment imprimé en français et mis en circulation. Il revient tous les ans pour être révisé, et le traducteur a ainsi l'occasion de modifier quelques bévues ayant échappé à sa vigilance.

Les promotions se font rapidement dans ce secteur dynamique où le traducteur commence à s'ennuyer après six ou douze mois d'exercice. Le genre des textes ne répond plus à ses aspirations secrètes; il se remet à lire les petites annonces des journaux, où, de fait, les offres d'emploi ont commencé à paraître. L'employeur demande tout modestement une personne connaissant le français et sachant et voulant traduire des textes intéressants. Le salaire est bon, puisqu'on l'engage, dès le départ à un niveau 
supérieur à celui d'un unilingue anglophone. Malgré cela, la recherche d'un tel employé est ardue. La compagnie met parfois six mois à trouver l'oiseau rare, qui, les premiers mois de contentement passés, se met à rêver d'un changement de décor. Comment garder un tel être instable et capricieux, qui se lance dans des arguments et des polémiques alors que les autres employés anglophones de l'étage sont silencieux et n'ont en fait aucune revendication. La routine de leur travail les tient occupés. Ceux-ci ne songent pas tellement à circuler d'un employeur à l'autre, pour gagner $5 \$$ de plus par semaine.

Les mois passent. Le nombre des documents à traduire faisant boule de neige, la direction est obligée de créer de nouveaux postes de traducteur, qu'elle arrive pourtant difficilement à combler. Toutes les compagnies ont commencé maintenant à comprendre la valeur d'avoir un (ou des) traducteur(s) sur les lieux mêmes. Plus économique, rapports suivis, liens amicaux établis, échanges de mots français/ anglais. C'est bon aussi pour créer de l'intérêt pour la langue française. Les collègues anglophones se sentent stimulés, intéressés. Il se forme, petit à petit, un îlot francophone où aboutissent tous les textes à traduire de toute la compagnie. Il n'est plus nécessaire ni d'envoyer les textes à traduire à Montréal, ni à les donner à des bureaux privés de traduction de la place, qui imposent des tarifs prohibitifs, puisqu'ils calculent le texte traduit au nombre de mots.

Le bruit courait à l'époque que le chef du personnel d'une compagnie avant-gardiste avait demandé à quelques agents d'immigration de l'aéroport d'appréhender tout immigrant parlant français, né de préférence à Paris, mais au pire des cas, dans les alentours de la Ville Lumière, et de le diriger immédiatement, pieds et poings liés, vers le poste qui l'attendait. A un moment donné, cette profession bourgeonnante vit se rallier à ses effectifs, des masseurs, des soudeurs, des maître queux, des mécaniciens, des vendeurs ambulants, dont le bagout et l'accent avaient hypnotisé l'interviewer émerveillé de tant de facilité verbale. À cette époquelà, il suffisait d'être beau parleur pour se dire traducteur. Les textes rédigés alors portent encore les stigmates de l'amateurisme, la marque d'une culture limitée.

Une autre source de recrutement était les jeunes filles qui venaient de Montréal ou de Québec à Toronto pour apprendre l'anglais. Elles se plaçaient dans des familles anglophones. Les quelques premiers mois passés à garder les enfants en bas âge leur donnaient à réfléchir et elles ne tardaient pas à se mettre à la recherche d'autres possibilités. Les compagnies étaient enchantées de les engager comme dactylos bilingues ou même traductrices junior. $\mathrm{Ce}$ - pendant, le travail de dactylographie étant exclusivement en français, elles commençaient, au bout de quelques mois, là encore, à chercher à changer leur sort. Elles étaient venues à Toronto pour apprendre l'anglais, or elles travaillaient en français toute la journée. Là aussi donc, roulement effréné. Les traducteurs ne savaient plus comment amadouer ces virtuoses de la machine. Grâce à elles, ils pouvaient prendre leur temps pour écrire et récrire les traductions. Vingt fois sur le métier, ils remettaient leur ouvrage. Le traducteur, après quelques mois d'exercice, assumait entièrement la responsabilité de son produit fini. Il se sentait à l'égal des actuaires et des avocats de son employeur. Il redressait l'échine. D'ailleurs, il ne l'avait jamais courbée. Dès le premier jour de son entrée en service, collègues anglophones et employeur lui avaient fait sentir son importance, et l'ampleur de son rôle. Certains diraient de sa mission, puisqu'il assurait le lien avec la province soeur, le Québec. Cependant, malgré tout, il arrivait que le néophyte lancé dans la traduction se lassait vite. Le style cassé des textes anglais, le manque d'outils, et d'orientation, lui donnaient du fil à retordre. Plus les postes de traducteurs se créaient, plus il y avait de polémiques. Chaque traducteur avait une version différente de la phrase anglaise. Et une version différente pour ce qui était de la tournure à adopter en français. Trop de marmitons commençaient à gâter la sauce. On voulait bien être accommodant. Mais on sentait soudain en soi l'urgence d'aller voir ailleurs. Ailleurs, cela voulait dire une autre compagnie, et à partir de 1968 , le Bureau des traductions du gouvernement fédéral ou bien un autre domaine. Il arrivait donc que traducteur et traduction se brouillaient à jamais. Certains praticiens, les hommes surtout, pensaient à s'établir à leur propre compte puisque cette pratique s'annonçait fructueuse, le domaine lucratif. On n'avait qu'à compter les mots. Ca chiffrait vite et gros. En ce temps-là, on se faisait payer un ou deux cents le mot; aujourd'hui, avec l'inflation, et la nature du texte, on peut exiger jusqu'à 30 cents le mot.

Donc, ouverture de cabinets de traductions, ou bien, si l'on voulait travailler solo, on devenait pigiste. Après les premiers piétinements, on démarrait et l'on s'établissait traducteur indépendant. Cela demande cependant un caractère particulier. N'est pas pigiste qui veut. Mais au pire des cas, on pouvait retourner chez l'employeur qui, il faut le dire, accueillait l'enfant prodigue à bras ouverts.

Les années qui passaient commençaient à consolider ce métier (cette profession). Celui qui se sentait à l'aise dans les recherches, la rédaction, les discussions, les controverses, voyait les choses prendre tournure de carrière puisque les promotions arrivaient rapidement. Il commençait alors à chercher à élargir ses horizons, à 
entrer en contact avec d'autres traducteurs de la place, ou d'autres villes (Montréal, Ottawa), se chercher des ouvrages de référence introuvables à Toronto et tout aussi inexistants à Montréal. Il fallait s'adresser aux libraires parisiens, geste qui impressionnait $e t$ les proches collègues (anglophones et francophones) et la direction, et se constituer un petit fichier de termes qui revenaient souvent.

Après enquête, et lecture approfondie des colonnes du quotidien "Le Devoir", on apprenait qu'à Montréal, il y avait aussi une activité traductionnelle, et même publication de documents intéressants. On apprenait ainsi qu'à Radio-Canada, qui publiait le "C'est-à-dire " en novembre 1960, venait de se constituer un comité de linguistique sous la présidence de $M$. Philippe Desjardins et parmi le nombre impressionnant de ses adjoints, on relevait le nom de Robert Dubuc, traducteur à Ottawa. Plus tard, d'autres noms qui: deviendront célèbres font leur apparition. Jean-Marie Laurence, grammairien et linguiste, Henry Bergeron. On apprend aussi qu'il existe de bons ouvrages, dont celui de Pierre Daviault, surintendant du Bureau des traductions du Secrétariat d'Etat, et celui de Vinay et Darbelnet, celui d'André Clas et de Paul Horguelin, d'Albert Baudet, etc. Il existe aussi une revue de traducteurs, Meta.

Vers la fin des années soixante, on apprend que M. Maurice Beaulieu va diriger à Québec un bureau qu'on a baptisé l'Office de la langue française, d'où l'on pourra se procurer gratuitement des cahiers de vocabulaires, notamment ceux sur les assurances, les anglicismes, les régionalismes de bon aloi, etc. Le tout commence à se concrétiser. Il y a du renfort qui arrive. Le praticien n'est plus laissé à ses seuls moyens. II s'épanouit. D'autres font leur entrée dans l'arène. Des professeurs commencent à se tourner vers la traduction. C'est un métier lucratif où il $\mathrm{y}$ a plein de débouchés, alors que leur profession est sursaturée.

Vers 1967, les structures sont déjà bien dessinées dans les services français des compagnies qui ont pris évidemment de l'ampleur. Quelques grosses compagnies, à l'heure actuelle, comptent une trentaine d'employés dans leurs services de linguistique. On remarquera que l'appellation change au fil des ans. De «services de traduction», ça devient «services français", puis «services de linguistique».

C'est vers cette date-là qu'un jour béni, une voix parlant français au téléphone demande à s'adresser au chef de service ou au chef traducteur qui a déjà sous ses ordres une bonne équipe composée dans un ordre hiérarchique intéressant de traducteurs secondée d'une bonne équipe de dactylos. La voix dit qu'il existe à Ottawa, depuis des années déjà, un groupement de traducteurs, connu d'abord sous le nom d'Association technologique de langue française
d'Ottawa, et fondé le 10 novembre 1920 . C'est l'aînée des sociétés de traducteurs du Canada. Son nom actuel d'Association des traducteurs et interprètes de l'Ontario (ATIO) lui a été conféré par lettres patentes supplémentaires le 10 septembre 1962. La voix ajoute que si jamais quelqu'un du bureau désire en faire partie, il doit présenter son dossier personnel, se faire accepter, et se présenter ensuite à l'hôtel Ford de la rue Dundas, quartier chinois de Toronto, pour passer les examens d'agrément.

Parallèlement à la montée du nationalisme au Québec, le gouvernement fédéral adopte en 1969 la Loi sur les langues officielles, d'où s'ensuit l'expansion du Bureau des traductions et sa décentralisation. Le traducteur fédéral se sert surtout d'un dictaphone, et le produit fini est soumis à des paramètres d'évaluation très sévères. Ainsi discipliné, le praticien acquiert une très grande vitesse, dictant parfois plus de cinq mille mots par jour, d'affilée, ce qui le rapproche de l'interprète simultané qui traduit à partir d'un texte qu'on lui a remis quelque temps avant que le conférencier ne commence à parler, avec la seule différence qu'il dispose d'ores et déjà d'un arsenal riche et varié d'ouvrages de référence et surtout des services de la Banque de terminologie. La Banque, créée en novembre 1974, a repris les travaux et le logiciel de la Banque de terminologie de l'Université de Montréal. Le Canada est lancé au premier rang mondial dans le domaine de la traduction.

La terminologie fait partie intégrante de la traduction. Sans avoir trouvé le mot juste, le traducteur ne peut pas dénouer le noud gordien, trancher et élucider le mystère que recèlent certains textes hermétiques. C'est pourquoi, à la simplicité d'attitude et de tempérament du traducteur des années 60 a succédé, au début des années 70 , un être plutôt renfermé, assez inquiet, égocentrique et exclusif, à quelques exceptions près. Je dirai même que quelques systèmes ont favorisé ce renfrognement de caractère. Alors qu'autrefois l'équipe des traducteurs s'asseyait coude à coude, par rangées de trois bureaux, se consultant constamment, échangeant des mots et des expressions, dans une atmosphère détendue et plaisante, chaque traducteur, désormais, ne cherche plus qu'à se terrer dans un coin, derrière un paravent, il ne cherche plus qu'à s'isoler. Les collègues ne se parlent plus. Tout contact intellectuel est coupé. Les machines plus perfectionnées, électriques et plus bruyantes des dactylos assises souvent à quelques pieds des traducteurs font un tintamarre d'enfer. Le traducteur a du mal à se concentrer sur son texte; il commence à mettre ses trouvailles terminologiques sous clef, il ne veut les communiquer à personne. Dans certains bureaux, quelques traducteurs travaillent de nouveau tres vite, maintenant, parce qu'ils ont su se constituer un petit fichier person- 
nel ultra-secret. L'avènement de la Banque de terminologie et de ses services de documentation (ces derniers ayant existé depuis toujours), va introduire un changement important. Maintenant, tout traducteur a accès en principe aux arcanes des termes introuvables. Il ne reste plus, pour se distinguer de ses collègues, qu'à se rabattre sur la technique et le style, soigner les tournures pour ne pas que le produit fini sente la traduction... Certains bureaux ont créé un nouveau poste, celui de "réviseur " qui ne s'occupera que de lire exclusivement le texte traduit, et de s'escrimer sur certaines phrases et tournures qu'il jugera barbares et hérétiques. La dissension ne tarde pas à s'installer... Les relations interpersonnelles sont tendues, parce que naguère, la personne chargée de lire les traductions, le faisait avec grand respect et doigté. De fait, il s'agissait d'une personne qui avait de la difficulté à écrire. Le traducteur se sentait le créateur, supérieur en quelque sorte. Lecteur et traducteur étaient sur un pied d'égalité à la rigueur. Là, par un revirement des événements et de la situation, le réviseur peut s'escrimer dans le texte du créateur. Les rapports se teintent de sadisme, de cruauté, et parfois aussi, il faut le dire, de masochisme. L'élaboration de la pensée, son développement et son exposition procédant directement du bagage culturel et des antécédents de l'auteur, on ne peut pas changer impunément l'ordre et la nature des éléments de la phrase. La discorde s'installe entre réviseur et révisé. Et la sarabande folle du début des années 60 recommence. Le traducteur se remet à lire les petites annonces d'offre d'emploi des journaux. Mais maintenant, les exigences sont tout autres: l'employeur demande l'appartenance à une société de traducteurs, un baccalauréat en traduction ou l'équivalent, de trois à cinq années d'expérience, dans un domaine précis et de l'entregent pour travailler harmonieusement et efficacement dans une équipe moderne et dynamique. On offre aussi maintenant des postes de terminologue, de recherchiste, ou de documentaliste. La Loi 101 du Québec a ouvert de très vastes horizons. Le traducteur n'est plus cet être taillable et corvéable à souhait. Donc en 1980 , le champ de la profession s'est élargi. Outre les sous-professions ainsi dérivées, le nombre des domaines à aborder s'est diversifié (assurances, finances, administration, médecine, technique, mécanique et le reste, sans parler de la météo, de l'informatique, de l'élec- tronique et de l'énergie). Les universités sont arrivées à la rescousse. Création de programmes dans presque toutes les universités des deux provinces sours (l'Ontario et le Québec). Aussi, parution d'ouvrages sur les différentes façons de manier cet art, ce métier ou cette technique. Là aussi les avis sont partagés. Diverses théories, critères et paramètres apparaissent et disparaissent. Maintenant, tout est là pour faire de cette pratique une profession bien établie. Lorsqu'on pense qu'elle existe depuis le temps de Babel et qu'on atermoie encore quelques millénaires plus tard sa reconnaissance. Toutes ses lettres de noblesse ne lui suffisent-elles pas?

On s'est plu à comparer d'abord cette pratique à celle de la médecine ou de la loi. Peut-être qu'il n'aurait fallu la rapprocher d'aucune autre profession et la considérer uniquement dans son intégralité. Mais nous ne sommes pas les seuls praticiens à désirer la reconnaissance professionnelle. Je lis dans les journaux de temps à autre, que tel groupement va augmenter ses pressions auprès du gouvernement pour se faire reconnaître.

Depuis 1967, l'ATIO a apporté au traducteur un soutien incommensurable. Elle poursuit la reconnaissance professionnelle, le perfectionnement des connaissances, les contacts avec les employeurs. Aujourd'hui, l'ATIO est en plein essor, après avoir connu des jours difficiles en 1975. A Toronto, dès 1976, M. Gérard Gauthier, le vice-président du Centre de l'Ontario, a regroupé les membres qui avaient commencé à se disperser et, en 1978, le premier congrès annuel tenu à Toronto a connu un franc succès. En 1979 , le professeur Brian Harris a inauguré les premiers ateliers mensuels de l'ATIO. L'ATIO veille aux intérêts du traducteur $e t$ du public ; elle s'intéresse aux débouchés d'emploi et contribue au perfectionnement des connaissances professionnelles. Elle collabore avec les universités et dialogue avec les employeurs.

Je vais conclure ce tour d'horizon en vous citant la phrase que j'ai retenue du bref discours de remerciement d'Ewald Osers de Grande-Bretagne, lauréat du Prix de la traduction de la FIT à Montréal en 1977: «Entre le traducteur et le texte qu'il traduit, il faut qu'il y ait un amour, je dirais un très grand amour $[. .$.$] sinon,$ il lui serait impossible d'endurer ce qu'il endure $[\ldots]$ »

Chake Tchilinguirian

Achevé d'imprimer en juin 1982 par les travailleurs des Ateliers Marquis de Montmagny. 